\title{
The impact of fine-tuning of optical recognition system on database reliability
}

\author{
P.A. Modesti ${ }^{\mathrm{a}, \mathrm{b}, *}$, L. Massetti ${ }^{\mathrm{a}}$, M. Bamoshmoosh ${ }^{\mathrm{c}}$, M. Baldereschi ${ }^{\mathrm{a}}$, G.E. Cambi ${ }^{\mathrm{a}}$, S. Rapi ${ }^{\mathrm{a}}$ \\ a Department of Critical Care Medicine, University of Florence, Florence, Italy \\ ${ }^{\mathrm{b}}$ Don Carlo Gnocchi Foundation, IRCCS Florence, Italy

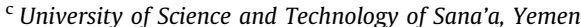

\section{A R T I C L E I N F O}

Article history:

Received 2 May 2011

Accepted 11 May 2012

\section{Keywords:}

Data entry

Automatic data processing

Optical readers

Data management system

Clinical trial

\begin{abstract}
A B S T R A C T
Although optical reading systems are important tools to transfer data from a paper form to electronic databases, the impact of system fine-tuning on the final error rate is not usually considered. At the end of a multi-step process involving paper form design training of operators, and fine-tuning procedure, the final rate of error can be reduced from $0.65 \%$ to $0.05 \%$. Fine-tuning should be introduced as a standard procedure while using optical reading systems.
\end{abstract}

(c) 2012 Elsevier Ltd. All rights reserved.

\section{Introduction}

The reliability of data transfer from paper forms (PF) to electronic databases is a crucial point of any clinical and epidemiological study. To assure fidelity of data transfer, manual data entry can be performed independently by two operators (double data entry, DDE) [1], which is however a time-consuming procedure. More recently, the use of optical reading systems (ORS) [2-4] has been introduced and is now spreading especially among organizations with defined document processing requirements, business rules and industry regulations. Cost is considered the main limitation in transferring this technology to the field of research, with the cost effectiveness ratio being usually assessed on the basis of the number of PFs to be processed. Although ORS enables reaching a final error rate in the same range of DDE [2-4], the final data quality is often considered as a static characteristic of the system. Paper quality, printing quality, form design and scanner quality are now well recognized factors importantly affecting final data quality. Paper crashes and shadow errors may be reduced by the use of paper heavier than $80 \mathrm{~g} / \mathrm{m}^{2}$ [5]. Print quality is especially important for form recognition, which is indeed markedly impaired by dark or light printing. Defining a paper drop out color is also an important factor since different scanners may have different appropriate colors [7]. Likewise the importance of the writing instrument

\footnotetext{
* Correspondence to: Department of Critical Care Medicine, University of Florence, Viale Morgagni 85, 50134 Florence, Italy. Tel.: +39 0557949376.

E-mail address: pamodesti@unifi.it (P.A. Modesti).
}

adopted to fill forms should be considered [7], especially when black and white laser scanners are used. Several authors also addressed the importance of form design, paying special attention to the distance between distinct marking areas [6]. The use of high quality scanner is finally recommended in order to avoid mechanical difficulties in pulling through multiple pages simultaneously and the possibility of dropping or mixing $[4,6]$.

The possible occurrence of systematic errors rising from software bugs, inadequate technical support, mechanical problems, or inadequate software setting, have been reported as often clusterized in particular days [10]. In these conditions the scanned data may be lost and the batch has to be rescanned $[4,6]$. Finally the human factor as a cause that affects data quality during ORS should not be forgotten $[4,6]$. However, no study reported the importance of system calibration, or the tuning procedure. In addition the characteristics of PF which might hamper optical reading are poorly investigated.

Therefore we specifically investigated the effects of system tuning on the final rate of errors, the test retest reliability of the reading system, the influence on our PFs of errors caused by shadow from fields marked on the other side of the paper, and which part of the form can be critical for optical mark recognition.

\section{Methods}

This study is part of the "HYpertension and Diabetes in Yemen" (HYDY) project, a population-based prevalence survey of cardiovascular risk factors with clinic and instrumental 
evaluations of over 12,000 subjects (aged 6 through 70 years) who were permanent residents in three geographic areas of Yemen (capital area, the inland, and a coastal area) purposively selected to represent different climatic, nutritional and orographic regions of Yemen. The study complies with the principles laid down in the Declaration of Helsinki and was approved by the Ethical Committee of the University of Science and Technology, Sana'a, Yemen. District leaders and local chiefs also consented to the survey. All subjects gave informed consent. Data collection was conducted at home by centrally trained survey teams composed of two investigators of the opposite gender. Subjects received two separate home-visits. During the first visit three blood pressure (BP) and pulse rate measurements were taken according to guidelines [9] with validated automatic devices [10]. Finger prick blood samples were obtained from fasting subjects $(>8 \mathrm{~h}$ ) to measure glucose, cholesterol, and triglycerides blood values with validated point of care instruments [11,12], and a urine dipstick test was performed [13]. HYDY questionnaire was derived from the WHO STEP programme [14]. Subjects were visited again within the next ten days for a second session of BP and glucose measurements. All measurements were reported on HYDY PF. System validation was performed using 400 PFs randomly selected among those collected during the study.

\subsection{Characteristics of HYDY PF}

The PF, designed by Recogniform (Recogniform Technologies S.p.A., Rende, CS, Italy), was structured as a booklet of 8 A4-size pages printed on 2 duplex A3-size papers. Each booklet was identified by a unique identification ID number printed and barcoded together with the page number on each page. A representative page of HYDY PF is reported in Fig. 1.

Each question was associated with a number of answer fields to be marked appropriately by the interviewer. Each field was associated with 1 out of 3 possible answer formats: single area to be blackened by the interviewer (M1, $n=81$ ) (i.e. proteins at dipstick test, Figure 1$)$; double area (M2, $n=37$ ) (i.e. fasting period, Fig. 1 ); triple area (M3, $n=39$ ) (i.e. reading 1 systolic 1 , Fig. 1). To limit user-interface-design errors, researchers were specifically trained on how to fill every single answer field.

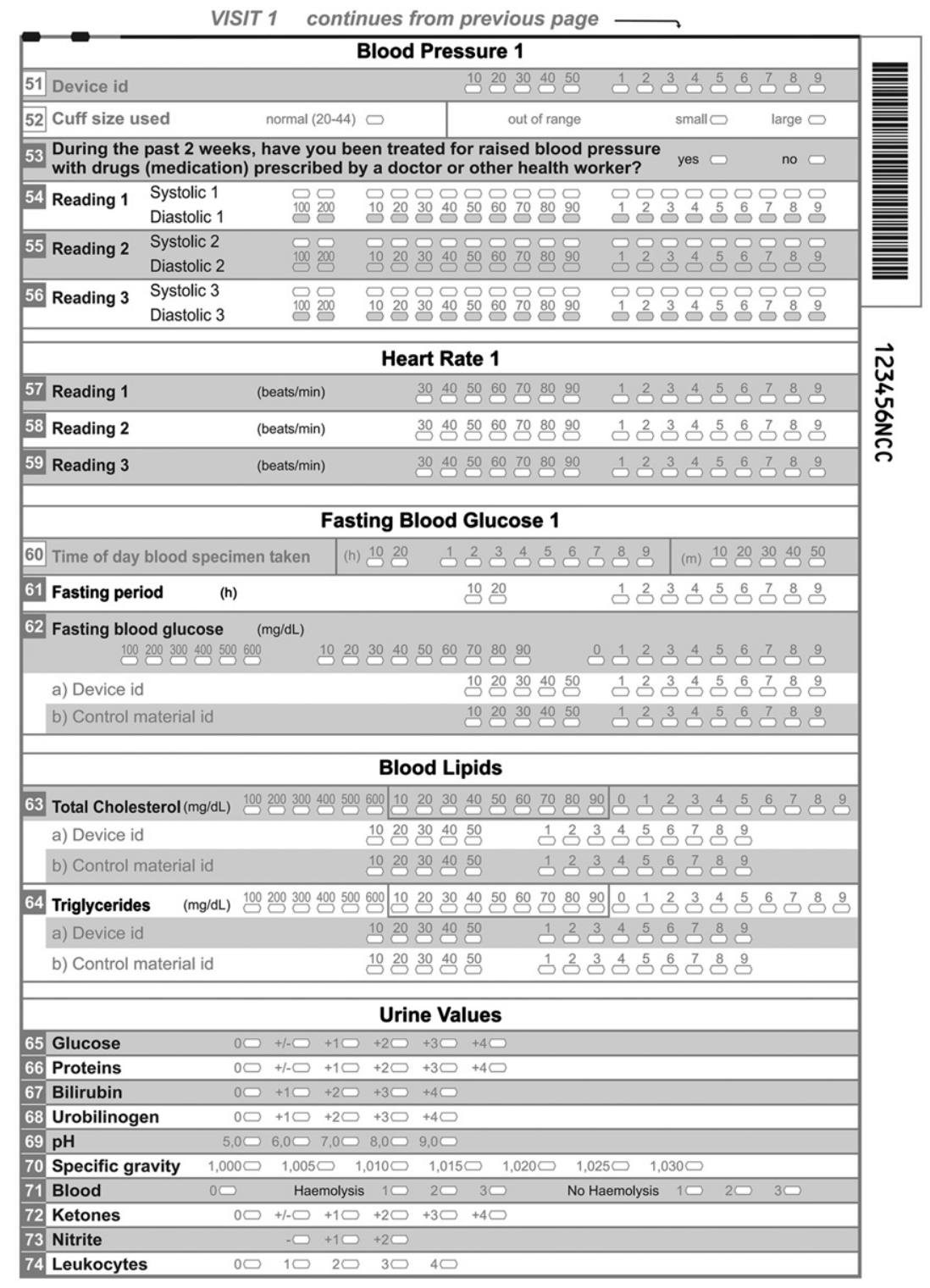

Fig. 1. Representative page of the HYDY paper form. 


\subsection{Form reading}

Optical reading questionnaires followed the following fourstep process activated by the operator in Sana'a (Yemen), through a user-friendly application developed by Microsoft Visual Basic.NET 8:

(a) Scan and pack: Filled PFs were read by the scanner (Fujitsu fi-5530C) and pages of each PF were merged by Recogniform in a single TIFF file.

(b) Preprocess: Recogniform verified PFs completeness.

(c) Validate: Recogniform verified the presence of a mark on mandatory fields (gender, age, district, village and interviewer-code). When no mark was found, the processing was stopped.

(d) Process: Recogniform verified Single mark only rules. In particular, in the presence of two or more, three or more, four or more areas for fields associated to a single (M1), double (M2) or triple (M3) the system proposes the form in a "Verifier windows" to be checked by the operator. If the operator was unable to solve the problem a -9999 code was reported in database.

Only PFs passing all the four steps were stored in the local database connected to the coordinating center in Florence (Italy).

\subsection{System calibration (Tuning)}

Two hundred randomly selected PFs (TIFF files) out of the total 400 , were preliminary read using default ink threshold (IT) setting (10\%). IT is the minimum percentage of the marking area that must be blackened to make the software detecting the presence of a mark.

The obtained database (DB-A) was manually checked against the original booklets by two HYDY investigators.

Errors were identified according to the following rules: (1) a value recorded in the DB-A with no value in the booklet (fake data); (2) no value recorded in the DB-A with a value in the booklet (unread data); (3) different value between DB-A and the booklet (discordant data); (4) -9999 code recorded in the DB-A with single mark in the booklet (fake double mark); (5) no single mark in the booklet with no -9999 code in the DB-A (unrecognized double mark).

According to these corrections a new database was created (DB-B). The 200 booklets were then repeatedly processed using different IT setting (10-3\%). Databases (DB-Ai) created at each reading iteration (i) were automatically checked against DB-B according to the same above mentioned rules. The setting that provided the lowest number of errors at between-readings comparisons was selected as the final setting (FS).

\subsection{Test retest investigation}

During system tuning a test retest reliability check was also performed running three PFs compiled by different interviewers to consider different mark sizes and pressure. Each PF was acquired for 15 times with three different ink thresholds (4\%, 7\% and $10 \%)$. A total of 45 readings for each threshold were obtained for inter-form reading variability and 2355 points (157 marks acquired for each form) were available for inter- mark variability check.

\subsection{Shadow errors}

Our choice in the form design was to use a $90 \mathrm{~g} / \mathrm{m}^{2}$ paper to avoid errors caused by shadow from fields marked on the other side. Anyway, the incidence of this kind of errors was evaluated studying in detail the answers in overlapping regions. In our PF 11 couple of questions had front to back overlapping and the errors observed in these answers were optically revised one by one.

\subsection{System validation}

All the 400 booklets were then processed using both IS and FS settings and stored in two separate databases (DB-C and DB-D respectively). DB-C was manually checked against the 400 original booklets by the same two investigators and a manually corrected database was created (DB-F). The three databases (DB-C, DB-D and DB-F) were then cross checked by an automated procedure. Cross discrepancies were checked and solved against TIFF files and a new standard (DB-G) was created. DB-G was then considered as the reference for error rate calculation.

\subsection{Statistical analysis}

Differences between rate of errors in databases were tested with Wilcoxon and expressed as Odd ratio (OR). Error rates in the three possible answer formats (M1, M2 or M3) were compared with Kruskal-Wallis test. $P$-values $<0.05$ were considered significant. Analyses were carried out with SPSS (version 17).

\section{Results}

\subsection{Data collection and TIFF creation}

Seven out of the total 400 PFs $(0.0175 \%)$ entered into the system by the same interviewers were aborted because of missing entry required fields (interviewer code, $n=3$; gender code, $n=1$; district code, $n=1$; village code, $n=2$ ). Data to fill missing codes were made available eventually and aborted PFs were read again successfully after correction. All 400 PFs were thus included in the analyses.

\subsection{System calibration}

The check performed by the two investigators on the first group of data (database DB-A, 200 subjects) obtained with the reading system at ink threshold $10 \%$ (initial setting, IS) allowed to detect 224 errors out of the 31,400 fields. Error rate significantly differed between fields requiring a single marking area (M1, 55 out of 16,$200 ; 0.34 \%$ ), double marking area (M2, 43 out of 7400 ; 0.58\%; OR 1.72: 95\% Cl 1.15-2.55), and triple marking area (M3, 126 out of 7800; 1.62\%; OR 4.82: 95\% Cl 3.51-6.62) ( $p=0.002$ at Kruskal-Wallis test). Iterative change of ink threshold (from $10-3 \%$ ) resulted in a significant progressive error rate reduction down to $0.05 \%$ at the ink threshold of $4 \%$ (OR $0.07 ; 95 \% \mathrm{Cl} 0.04-$ 0.12 vs. IS) (Fig. 2). Ink threshold of $4 \%$ was thus considered as the final setting (FS).

\subsection{Test retest investigation}

In repeated readings no inter-form or inter-mark variability were found with ink threshold values of $4 \%$ (FS) or $7 \%$. With ink threshold value of $10 \%$ (IS), 24 out of 45 (53.3\%) readings showed each one a single discrepancy, resulting in an overall $1 \%$ discrepancy rate (24 out of $2355,1 \%$ ). 


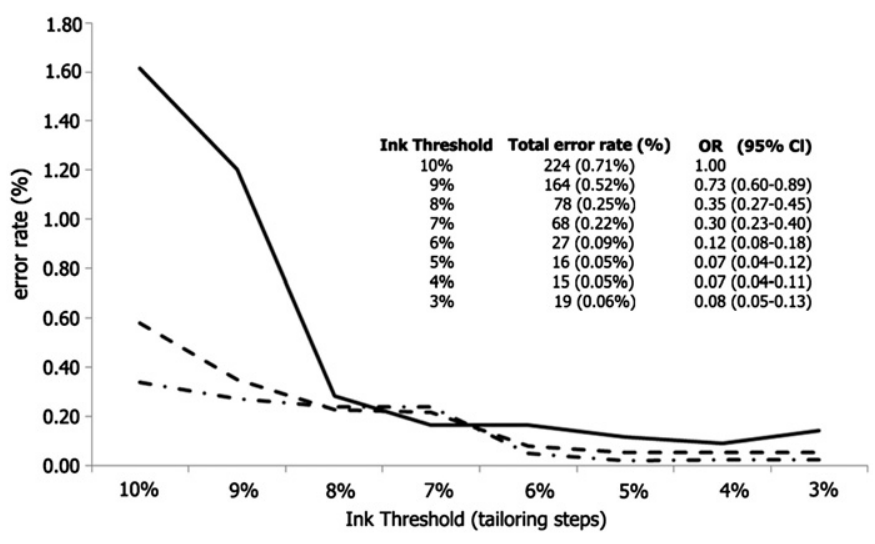

Fig. 2. Rate of error for answer type M1 (dashed-dotted line), M2 (dashed line) and M3 (thick line) for each tuning step. Total error rate and odd ratio vs initial setting (10\%) for each tuning step are reported in the insect.

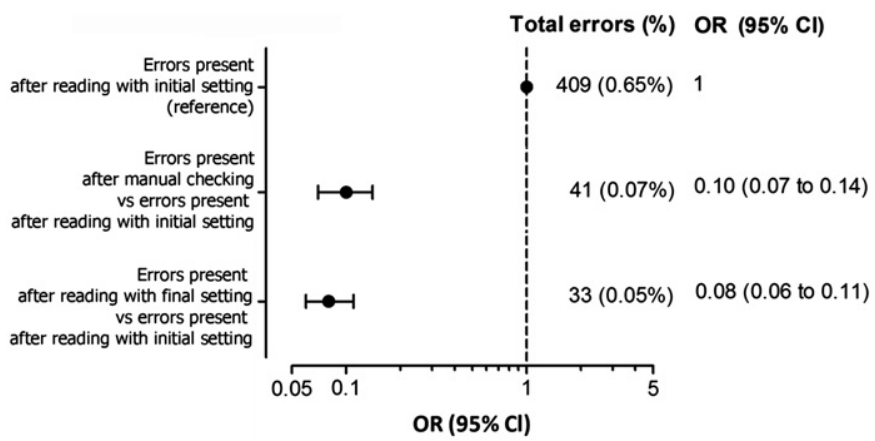

Fig. 3. Odd ratios for the rate of error reduction after manual correction (rate of error in DB-F) and after reading the same TIFF type files with final setting (DB-D) vs. rate of error present on database after reading with initial setting (DB-C). Rate of errors is calculated vs. reference database (DB-G).

\subsection{Shadow errors}

A total of 5 errors in the 400 PFs were found in the 24 overlapped questions $(0.052 \%)$. This rate was not different from that of non-overlapped questions (28 out of $133 ; 0.053 \%$ ).

\subsection{Error rate with final setting}

The database obtained by reading the 400 TIFF files (DB-C) with IS carried an error rate after manual checking comparable to the DB-A. Odds ratios for the rate of error reduction after manual checking (rate of error in DB-F) and after reading the same TIFF type files with FS (DB-D) vs. rate of error on database after reading them with IS (DB-C) are reported in Fig. 3. The error rate with FS $(0.05 \%)$ did not significantly differ from the rate obtained with manual checking $(0.07 \%$; OR $1.24 ; 0.79-1.97, p=0.526$ at Wilcoxon signed ranks test).

Using FS automated reading, data inconsistency turned out to be the most frequent type of error (17 out of 33), followed by fake data errors $(n=8)$, unread data errors $(n=4)$ and fake double errors $(n=4)$, while no "single mark only" error was found. The M1 answer format had also lower error rate (0.02\%) than M2 (0.07\%) and M3 (0.09\%, $p=0.008$ Kruskal-Wallis test).

\section{Discussion}

Single manual data entry has been reported to be associated with a final rate of error [3,16-21] ranging between $0.11 \%$ [3] and
$0.68 \%$ [17]. Although the final error rate of double data entry (DDE) is lower than $0.1 \%[3,16]$, the procedure is time consuming and conflicting perspectives were expressed regarding the value of DDE in clinical trials $[1,19,20,22,23]$. The use of ORS instead of the DDE in large epidemiological studies may offer some important advantages in terms of time and cost savings and standardized data management and quality.The chance for errors in clinical research databases during data entry is highly recognized [15]. The rate of errors is related to the specific method and should be investigated in detail before data acquisition. A well setted ORS seems to offer the opportunity to reach a final accuracy comparable to DDE, the rate of error with the use of Teleform [3,4,21] ranging between $0.04 \%$ [4] and $0.07 \%$ [3], a single study performed with a system developed in Formic reporting an error rate of $0.02 \%$ [2]. A $0.1 \%$ absolute rate of error is usually considered as acceptable cut-point [24].

A proper design of the recognition paper form and an adequate training of the operators are essential requirements to ensure good data quality [4-8]. In particular a proper form design is crucial for the investigator and the recovery system. Paper thickness heavier than $80 \mathrm{~g} / \mathrm{m}^{2}$ and avoiding overlapping fields play a major role in reducing shadow errors. The possibility of dropping pages belonging to the same PF or mixing pages belonging to different PFs can be avoided by identifying pages with a barcode; the system checks for the completeness of the PF and if one of the pages is missing the process stops and the PF must be scanned again. Therefore, form structure is to be considered in the phase of study design. While designing HYDY study, all these points had been carefully considered, yielding no PF mismatch in our database. The present study however shed light on a different aspect in form design that is the importance of the number of markings used to encode a variable. Fields linked to a single (M1, i.e. Yes or No), a double (M2, i.e. day or month), or a triple marking area (M3, i.e. blood pressure values), may indeed be associated with a different rate of error.

The present study, besides reporting an accuracy value comparable to previous observations, shed light on the value of a crucial methodological issue, the importance of tuning ORS and suggests this procedure as a preliminary necessary step to any computerized control [25]. To our knowledge no study was focused on clarifying the importance of tuning the reading system to improve precision and accuracy. The system calibration we adopted resulted in an over 10 fold improvement of accuracy (error rate reduction from $0.65 \%$ to $0.05 \%$ ). IT value reduction from $10 \%$ to $3 \%$ paralleled a progressive reduction of unread data. However, below the value of $4 \%$ the number of fake error started increasing, with a resulting increase of total errors (Fig. 2). The optimum IT is thus to be searched with a specific tuning procedure because it may depend on the marking area and form design. Results may vary with different PF.

Guidelines on the application of new technology to population data collection and capture have been provided [26]. In medical settings, such as in blood donation and transfusion service, (1) information credibility, (2) information integrity, (3) information synergy, and (4) information security, have to be respected [27]. The guidance to assist manufacturers and users in developing a computer system validation programs provided by Food and Drug Administration (FDA) [28] suggests system validation in the user's facility to assure suitability for specific operations and workload to meet required specific needs. The level of confidence and, therefore, the level of validation effort needed, vary depending upon the potential hazards posed by the automated functions of the system.

It is now more popular to collection information by mobile devices, e.g., tablet or ipad. Newer technologies adopting a Mobile/Handheld device based data collection techniques have some advantages over OMR technology [29]. More precisely in 
case of a mobile based data collection data collected in the field is accessible to a large group of people immediately (turnaround time). Conversely with OMR technology data is accessible only after all the forms are physically converged to a centralized scanning unit. OMR can be used only as a standalone data collection tool unlike various emerging technologies like GPS, Mobile applications (versatility). OMR sheets have to be carefully handled as any damage on the sheet will lead to errors (damaging of OMR sheet). OMR logic does not accommodate any error validation logic and any wrong entry cannot be proof-read immediately (error). Finally it is not easy to make changes once the OMR template is already designed (rigidity).

When mobile healthcare radiofrequency (RF) wireless technology is used, electromagnetic interference (EMI) has also to be considered [30]. In addition, data and signal disruption and slowdown, coexistence problems with other wireless technology [31], and security breaches $[32,33]$ are significant risks for the safe and effective use of wireless technology in medical devices and device systems. Key risks related to the use of wireless technology specifically considered by FDA, Center for Devices and Radiological Health for wireless medical devices and systems [34] are quality of service (QoS), data integrity (e.g., data throughput, latency, corruption), coexistence with other wireless equipment, security, and electromagnetic compatibility (EMC) [34].

In conclusion, an absolute rate of error lower than the acceptable cut-point of $0.1 \%$ can be reached at the end of a multi-step process involving recognition form design, operator training, and fine tuning of ORS.

\section{Summary}

Although optical reading systems (ORS) are useful tools to transfer data from paper forms (PF) to electronic database, clinical researchers may be unaware that the system may represent a direct source of errors in final database, and the impact of system fine-tuning on the final error rate is not usually considered. Present investigation was performed within the frame of HYDY study to measure error rate introduced in the final database by an ORS. The impact of fine-tuning, the relationship between error rate and the number of areas to be blackened for each field, shadow errors, and test retest variability, were investigated.

Ink threshold changes from $10 \%$ (default setting) to $4 \%$ (final setting) reduced the error rate from $0.65 \%$ to $0.05 \%(p<0.001)$, comparable to the rate achieved by visual checking $(0.07 \%$; OR $1.24 ; 0.79-1.97 ; p=0.526)$. Error rate was affected by the number of areas to be blackened in the form to fill a single field $(0.02 \%$, $0.07 \%$ and $0.09 \%$ for single, double and triple area respectively, $p=0.008)$. The final error rate did not differ between overlapped and non-overlapped questions on paper forms ( $0.05 \%$ for both). In repeated readings no inter-form or inter-mark variability were found when ink threshold values was $<7 \%$.

Fine-tuning should be introduced as a standard procedure while using optical reading systems to reach the acceptable cutpoint $(0.1 \%)$ of error rate.

\section{Conflict of Interest Statement}

None declared.

\section{Acknowledgments}

HYDY Study was funded by the Ministero dell'Università e della Ricerca (Direzione Generale per le strategie e lo sviluppo dell'internazionalizzazione della ricerca scientifica e tecnologica, Rome, Italy) within the frame of the Executive Programme of Scientific and Technological Cooperation between Italy and Yemen (Grant 269/P/0116202 to PA Modesti), with additional support by Menarini International Operations Luxembourg SA. Funding bodies had no role in the study design, in the collection, analysis and interpretation of data, in the writing of the manuscript, or in the decision to submit the manuscript for publication. The collaboration of L. Bottaro is gratefully acknowledged.

\section{References}

[1] J.D. Neaton, A.G. Duchene, K.H. Svendsen, D. Wentworth, An examination of the efficiency of some quality assurance methods commonly employed in clinical trials, Stat. Med. 9 (1990) 115-124.

[2] E.T. Smyth, G. Mcilvenny, J.G. Barr, L.M. Dickson, I.M. Thompson, Automated entry of hospital infection surveillance data, Infect. Control Hosp. Epidemiol. 18 (1997) 486-491.

[3] C.K. Jorgensen, B. Karlsmose, Validation of automated forms processing a comparison of teleform with manual data entry, Comput. Biol. Med. 28 (1998) 659-667

[4] C. Jinks, K. Jordan, P. Croft, Evaluation of a computer-assisted data entry procedure (including teleform) for large-scale mailed surveys, Comput. Biol. Med. 33 (2003) 425-437.

[5] B.A. Mortagy, N.N. Hegazy, Preparation for Egypt's population and housing census. Data processing challenges, Afr. Stat. J. 1 (2005) 104-113.

[6] P. Guerette, B. Robinson, W.P. Moran, C. Messick, M. Wright, J. Wofford, et al., Teleform TM scannable data entry: an efficient method to update a community-based medical record? Proc. Annu. Symp. Comput. Appl. Med. Care (1995) 86-90.

[7] J.S. Shapiro, M.J. Bessette, K.M. Baumlin, D. Fish Ragin, L.D. Richardson, Automating research data collection, Acad. Emergency Med. 11 (2004) 1223-1228.

[8] S.M. Downs, A.E. Carroll, V. Anand, P.G. Biondich, Human and System Errors, Using Adaptive Turnaround Documents to Capture Data in a Busy Practice, AMIA Annual Symposium Proceedings, 2005, pp. 211-215.

[9] G. Mancia, G. De Backer, A. Dominiczak, R. Cifkova, R. Fagard, G. Germano, et al., The task force for the management of arterial hypertension of the European Society of Hypertension, the task force for the management of arterial hypertension of the European Society of Cardiology, 2007 guidelines for the management of arterial hypertension: the task force for the management of arterial hypertension of the European Society of Hypertension (ESH) and of the European Society of Cardiology (ESC), Eur. Heart J. 28 (2007) 1462-1536.

[10] G.S. Stergiou, N.G. Yiannes, V.C. Rarra, Validation of the Omron 705 IT oscillometric device for home blood pressure measurement in children and adolescents: the Arsakion school study, Blood Press Monit. 11 (2006) 229-234.

[11] I. Püntmann, W. Wosniok, R. Haeckel, Comparison of several point-of-care testing (POCT) glucometers with an established laboratory procedure for the diagnosis of type 2 diabetes using the discordance rate. A new statistical approach, Clin. Chem. Lab. Med. 41 (2003) 809-820.

[12] S. Rapi, C. Bazzini, C. Tozzetti, V. Sbolci, P.A. Modesti, Point-of-care testing of cholesterol and triglycerides for epidemiologic studies: evaluation of the multicare-in system, Transl. Res. 153 (2009) 71-76.

[13] S. Rapi, L. Bartolini, D. Puliti, G.E. Cambi, M. Bamoshmoosh, M. Baldereschi, L. Massetti, et al., Conventional dipsticks in the screening of microalbuminuria and urinary tract infections killing 2 birds with one stone? Saudi Med. J. 31 (2010) 708-709.

[14] WHO STEP Programme. 〈www.who.int/chp/steps/en/〉, 2011 (accessed 16.05.11).

[15] S.I. Goldberg, A. Niemierko, A. Turchin, Analysis of data errors in clinical research databases, AMIA Annu. Symp. Proc. 6 (2008) 242-246.

[16] M. Kawado, S. Hinotsu, Y. Matsuyama, T. Yamaguchi, S. Hashimoto, Y. Ohashi, A comparison of error detection rates between the reading aloud method and the double data entry method, Controlled Clin. Trials 24 (2003) 560-569.

[17] J.E. Hilner, A. McDonald, L. Van Horn, C. Bragg, B. Caan, M.L. Slattery, et al. Quality control dietary data collection in the CARDIA study, Controlled Clin. Trials 13 (1992) 156-169.

[18] G.J. Prud'homme, P.L. Canner, J.A. Cutler, Quality assurance and monitoring in the hypertension prevention trial, Controlled Clin. Trials 10 (1989) 84S-94S

[19] R.A. Reynolds-Haertle, R. McBride, Single vs. double data entry in CAST, Controlled Clin. Trials 13 (1992) 487-494.

[20] D. Gibson, A.J. Harvey, V. Everett, M.K. Parmar, Is double data entry necessary? The CHART trials. CHART steering committee. Continuous, hyperfractionated, accelerated radiotherapy, Controlled Clin. Trials 15 (1994) 482-488.

[21] M.M. Wahi, D.V. Parks, R.C. Skeate, S.B. Goldin, Reducing errors from the electronic forms: a research data case study transcription of data collected on paper, J. Am. Med. Inf. Assoc. 15 (2008) 386-389. 
[22] R.A. Kronmal, K. Davis, L.D. Fisher, R.A. Jones, M.J. Gillespie, Data management for a large collaborative clinical trial (CASS: Coronary Artery Surgery Study), Comput. Biomed. Res. (1978) 553-556.

[23] S. Day, P. Fayers, D. Harvey, Double data entry: what value, what price? Controlled Clin. Trials 19 (1998) 15-24

[24] K. Kleinman, Adaptive double data entry: a probabilistic tool for choosing which forms to reenter, Controlled Clin. Trials 22 (2001) 2-12.

[25] D. Mauri, V. Karampoiki, J. Mauri, K. Kamposioras, G. Alexiou, G. Ferentinos, et al., SESy Europe task force, double-blind control of the data manage doesn't have any impact on data entry reliability and should be considered as an avoidable cost, BMC Med. Res. Methodol. 8 (2008) 66.

[26] United Nations Economic and Social Commission for Asia and the Pacific (ESCAP), Guidelines on the Application of New Technology to Population Data Collection and Capture. 〈www.unescap.org/stat/pop-it/pop-guide/ >, 2011 (accessed 14.12.11).

[27] B.N. Li, S. Chao, M.C. Dong, SIBAS: a blood bank information system and its 5-year implementation at Macau, Comput. Biol. Med. 37 (2007) 588-597.

[28] General Principles of Software Validation, Final Guidance for Industry and FDA Staff, January 11, 2002. 〈www.fda.gov/cdrh/comp/guidance/938.html 〉 2012 (accessed 07.05.12)
[29] D. Sabyasachi, IKP Centre for Technologies in Public Health, Optical Mark Recognition Technology for Rural Health Data Collection, November 2010. <www. ictph.org.in/downloads/Optical\%20Mark\%20Recognition\%20Technology\%20for\%20 Rural\%20Health\%20Data\%20Collection.pdf >, 2011 (accessed 14.12.11).

[30] D.H. Hoglund, Wireless and broadband management for integrated healthcare network, IT Horizons (2008) 39-45.

[31] R. Morrow, Wireless Network Coexistence, McGraw-Hill, 2004.

[32] International Electrotechnical Commission. Medical Electrical Equipment-Part 1: General Requirements for Safety. 2. Collateral Standard: Electromagnetic Compatibility-Requirements and Tests, IEC 60601-1-2:2001 and Amendment 1:2004, Geneva (Switzerland), IEC, 2001/2004

[33] International Organization for Standardization. Implants for Surgery-Active Implantable Medical Devices_Part 1: General Requirements for Safety, Marking and for Information to be Provided by the Manufacturer, ISO 14708-1:2000, Geneva (Switzerland), ISO, 2000.

[34] Food and Drug Administration, Draft Guidance for Industry and FDA Staff Radio-Frequency Wireless Technology in Medical Devices. 〈www.fda.gov/ MedicalDevices/DeviceRegulationandGuidance/GuidanceDocuments/ ucm077210.htm >, 2012 (accessed 07.05.12) 